\title{
THE TWO DIMENSIONAL GENERALIZED WEYL FRACTIONAL CALCULUS AND SPECIAL FUNCTIONS
}

\author{
V.B.L. CHAURASIA AND MUKESH AGNIHOTRI
}

\begin{abstract}
The object of this present paper is to derive a relation between the two dimensional I-transform involving a general class of polynomials and the Weyl type two dimensional Saigo operators of fractional integration. The results derived here are general in nature and include the results given earlier by Saigo, Saxena and Ram [10],Saxena and Ram [8], Saxena and Kiryakova [9] and Chaurasia and Srivastava [12].
\end{abstract}

\section{Introduction}

A. K. Rathie [11] introduced the I-function in the form

$$
\begin{aligned}
I(z) & =I_{p, q}^{m, n}[z]=I_{p, q}^{m, n}\left[z \mid \begin{array}{l}
\left(\alpha_{1}, A_{1}, a_{1}\right), \ldots,\left(\alpha_{p}, A_{p}, a_{p}\right) \\
\left(\beta_{1}, B_{1}, b_{1}\right), \ldots,\left(\beta_{q}, B_{q}, b_{q}\right)
\end{array}\right] \\
& =\frac{1}{2 i \pi} \int_{-i \infty}^{i \infty} \theta(\xi) z^{\xi} d \xi
\end{aligned}
$$

where

$$
\theta(\xi)=\frac{\prod_{j=1}^{m}\left\{\Gamma\left(\beta_{j}-B_{j} \xi\right)\right\}^{b_{j}} \prod_{j=1}^{m}\left\{\Gamma\left(1-\alpha_{j}+A_{j} \xi\right)\right\}^{a_{j}}}{\prod_{j=m+1}^{q}\left\{\Gamma\left(1-\beta_{j}+B_{j} \xi\right)\right\}^{b_{j}} \prod_{j=n+1}^{p}\left\{\Gamma\left(\alpha_{j}-A_{j} \xi\right)\right\}^{a_{j}}},
$$

where $a_{j}, j=1, \ldots, p$ and $b_{j}, j=1, \ldots, q$ are not, in general positive integers. Clearly for non-integral values of $a_{j}$ or $b_{j},(1)$ is not expressible as an H-function. Here $z$ may be real or complex but is not equal to zero and an empty product is interpreted as unity; $p, q, m$ and $n$ are integers such that $0 \leq m \leq q ; 0 \leq n \leq p, A_{j}>0(j=1, \ldots, p)$, $B_{j}>0(j=1, \ldots, q) ; \alpha_{j}, j=1, \ldots, p$ and $\beta_{j}, j=1, \ldots, q$ are complex parameters. The contour in (1) is presumed to be the imaginary axis $\operatorname{Re}(\xi)=0$ which is suitable indented in order to avoid the singularities of the gamma functions and to keep these

Corresponding author: V.B.L. Chaurasia.

Received October 3, 2008.

Key words and phrases. I-transform, Weyl type Saigo operator, fractional integration. 
singularities at appropriate side. For $a_{j}$ not an integer, the pole of the gamma functions of the numerator of (2) is converted to branch point. The branch cuts can be chosen in order that the path of integration can be distorted for the contour $\operatorname{Re}(\xi)=0$ as long as there is no coincidence of poles from $\Gamma\left(\beta_{j}-B_{j} \xi\right)$ and $\Gamma\left(1-\alpha_{j}-a_{j} \xi\right)$. The sufficient conditions for the absolute convergence of the contour integral (1) is given by

$$
\Omega=\sum_{j=1}^{m}\left|b_{j} B_{j}\right|+\sum_{j=1}^{n}\left|a_{j} A_{j}\right|-\sum_{j=m+1}^{q}\left|b_{j} B_{j}\right|-\sum_{j=n+1}^{p}\left|a_{j} A_{j}\right|>0 .
$$

This condition provides exponential decay of the integrand in (1), and region of absolute convergence of (1) is

$$
|\arg (z)|<\frac{\Omega \pi}{2}
$$

Srivastava [2] introduced the general class of polynomials

$$
S_{n}^{m}[x]=\sum_{k=0}^{\left[\frac{n}{m}\right]} \frac{(-n)_{m k}}{k !} A_{n, k} x^{k}, \quad n=0,1,2, \ldots,
$$

where $m$ is an arbitrary positive integer and the coefficients $A_{n, k}(n, k \geq 0)$ are arbitrary constants, real or complex. By suitable specialization of the coefficients $A_{n, k}$, the general class of polynomials can be reduced to large spectrum of polynomials as cited in the papers referred to above.

\section{Fractional Integrals and Derivatives}

An interesting and useful generalization of both the Riemann - Liouville and Erdelyi - Kober fractional integration operators is introduced by M. Saigo [5], [6] in terms of Gauss's hypergeometric function as given below. Let $u, v$ and $w$ are complex numbers and let $x \in R_{+}=(0, \infty)$ following [5], [6] the fractional integral $(\operatorname{Re}(u)>0)$ and derivative $(\operatorname{Re}(u)<0)$ of the first kind of a function $f(x)$ on $R_{+}$are defined respectively in the forms

$$
\begin{aligned}
I_{0, x}^{u, v, w} f & =\frac{x^{-u-v}}{\Gamma(u)} \int_{0}^{x}(x-s)^{u-1}{ }_{2} F_{1}\left(u+v ;-w ; u ; 1-\frac{s}{x}\right) f(s) d s ; \quad \operatorname{Re}(u)>0 \\
& =\frac{d^{n}}{d x^{n}} I_{0, x}^{u+n, v-n, w-n} f ; \quad 0 \leq \operatorname{Re}(u)+n \leq 1, \quad(n=1,2,3, \ldots) .
\end{aligned}
$$

where ${ }_{2} F_{1}(\rho ; \sigma ; \eta ; \cdot)$ is Gauss's hypergeometric function. The fractional integral $(\operatorname{Re}(u)>$ $0)$ and derivative $(\operatorname{Re}(u)<0)$ of the second kind are given by

$$
\begin{aligned}
J_{x, \infty}^{u, v, w} f & =\frac{1}{\Gamma(u)} \int_{x}^{\infty}(s-x)^{u-1} s_{2}^{-u-v} F_{1}\left(u+v,-w ; u ; 1-\frac{x}{s}\right) f(s) d s ; \quad \operatorname{Re}(u)>0,(8) \\
& =(-1)^{n} \frac{d^{n}}{d x^{n}} J_{x, \infty}^{u+n, v-n, w} f ; \quad 0 \leq \operatorname{Re}(u)+n \leq 1, \quad(n=1,2,3, \ldots) .
\end{aligned}
$$


The Riemann-Liouville, Weyl and Erdelyi-Kober fractional calculus operators follow as special cases of the operators $I$ and $J$ as detailed below.

$$
\begin{aligned}
R_{0, x}^{u} f & =I_{0, x}^{u, u, w} f=\frac{1}{\Gamma(u)} \int_{0}^{x}(x-s)^{u-1} f(s) d s ; \quad \operatorname{Re}(u)>0, \\
& =\frac{d^{n}}{d x^{n}} R_{0, x}^{u+n} f ; \quad 0 \leq \operatorname{Re}(u)+n \leq 1, \quad(n=1,2,3, \ldots) . \\
W_{x, \infty}^{u} f & =W_{x, \infty}^{u,-u, w} f=\frac{1}{\Gamma(u)} \int_{x}^{\infty}(s-x)^{u-1} f(s) d s ; \quad \operatorname{Re}(u)>0, \\
& =(-1)^{n} \frac{d^{n}}{d x^{n}} W_{x, \infty}^{u+n} f ; \quad 0 \leq \operatorname{Re}(u)+n \leq 1, \quad(n=1,2,3, \ldots) . \\
E_{0, x}^{u, w} f & =I_{0, x}^{u, 0, w} f=\frac{x^{-u-w}}{\Gamma(u)} \int_{0}^{x}(x-s)^{u-1} s^{w} f(s) d s ; \quad \operatorname{Re}(u)>0, \\
K_{x, \infty}^{u, w} f & =J_{x, \infty}^{u, 0, w} f=\frac{1}{\Gamma(u)} \int_{x}^{\infty}(s-x)^{u-1} s^{-u-w} f(s) d s ; \quad \operatorname{Re}(u)>0 .
\end{aligned}
$$

Following Miller [3] we denote by $S_{1}$ the class of functions $f(x)$ on $R_{+}$, which are infinitely differentiable with partial derivatives of any order behaving as $o\left(|x|^{-\gamma}\right)$ when $x \rightarrow \infty$ for all $\gamma$. Similarly we denote $S_{2}$, the class of functions $f(x, y)$ on $R_{+} \times R_{+}$, which are infinitely differentiable with partial derivatives of any order behaving as $o\left(|x|^{-\gamma_{1}},|y|^{-\gamma_{2}}\right)$ when $x \rightarrow \infty, y \rightarrow \infty$ for all $\gamma_{i}(i=1,2)$. The two dimensional Saigo operator of Weyl type fractional integration of order $\operatorname{Re}(u)>0, \operatorname{Re}(\eta)>0$ is defined in the class $S_{2}$ by

$$
\begin{aligned}
J_{x, \infty}^{u, v, w} J_{y, \infty}^{\eta, \beta, \alpha}[f(x, y)]= & \frac{x^{v} y^{\beta}}{\Gamma(u) \Gamma(\eta)} \int_{x}^{\infty} \int_{y}^{\infty}(t-x)^{u-1}(c-y)^{\eta-1} t^{-u-v} c^{\eta-\beta} \\
& { }_{2} F_{1}\left(u+v,-w ; u ; 1-\frac{x}{t}\right){ }_{2} F_{1}\left(\eta+\beta,-\alpha ; \eta ; 1-\frac{y}{c}\right) F(t, c) d t d c,
\end{aligned}
$$

where $v, \beta, w, \alpha$ are real numbers. More generally, a Saigo operator of Weyl type fractional calculus in two variables is defined by the differ integral expression

$$
\begin{aligned}
J_{x, \infty}^{u, v, w} J_{y, \infty}^{\eta, \beta, \alpha}[f(x, y)]= & \frac{(-1)^{M+N} x^{v} y^{\beta}}{\Gamma(u+M) \Gamma(\eta+N)} \frac{\partial^{M+N}}{\partial x^{M} \partial y^{N}} \int_{x}^{\infty} \int_{y}^{\infty}(t-x)^{u-1}(c-y)^{\eta-1} t^{-u-v} c^{-\eta-\beta} \\
& { }_{2} F_{1}\left(u+v,-w ; u ; 1-\frac{x}{t}\right){ }_{2} F_{1}\left(\eta+\beta,-\alpha ; \eta ; 1-\frac{y}{c}\right) F(t, c) d t d c, \quad(17)
\end{aligned}
$$

for arbitrary real $u$ and $\eta, M, N=0,1,2, \ldots$ For $f(x, y) \in S_{2}$ this differ integral exists and also belongs to $S_{2}[3]$.

In particular, if $\{\operatorname{Re}(u)+M\}>0,\{\operatorname{Re}(\eta)+N\}>0$ then (17) yield partial fractional derivative of $f(x, y)$.

On the other hand if we set $v=\beta=0,(17)$ yield the Weyl type Erdelyi-Kober operators in two dimensions

$$
K_{x, \infty}^{u, w} K_{y, \infty}^{\eta, \alpha}[f(x, y)]=J_{x, \infty}^{u, 0, w} J_{y, \infty}^{\eta, 0, \alpha}[f(x, y)]
$$




$$
\begin{array}{r}
=\frac{(-1)^{M+N} x^{w} y^{a}}{\Gamma(u+M) \Gamma(\eta+N)} \frac{\partial^{M+N}}{\partial x^{M} \partial y^{N}} \int_{x}^{\infty} \int_{y}^{\infty}(t-x)^{u+M-1}(c-y)^{\eta+N-1} \\
t^{-u-w} c^{-\eta-\alpha} F(t, c) d t d c .
\end{array}
$$

\section{Two-dimensional Laplace transform and I-transforms involving a general class of polynomials}

The Laplace transform $\mu(a, b)$ of function $f(x, y) \in S_{2}$ is defined

$$
\mu(a, b)=L[f(x, y) ; a, b]=\int_{0}^{\infty} \int_{0}^{\infty} e^{-a x-b y} f(x, y) d x d y
$$

where $\operatorname{Re}(a)>0, \operatorname{Re}(b)>0$.

Analogously, the Laplace transform of

$$
f\left(d \sqrt{x^{2}-h^{2}} H(x-h), f \sqrt{y^{2}-g^{2}} H(y-g)\right], \quad x>h>0, y>g>0
$$

and $H(s)$ denotes Heaviside's unit step function.

Definition. By two -dimensional I-transform $\Delta(a, b)$ involving a general class of polynomials of a function $F(x, y)$, we mean the following repeated integral involving two different I-functions with a general class of polynomials

$$
\begin{aligned}
\Delta(a, b)= & \Delta_{p, q, n_{1} ; p^{\prime}, q^{\prime}, n_{2}}^{m, n, m_{2}}[F(x, y) ; \rho, \sigma ; a, b] \\
= & \int_{h}^{\infty} \int_{g}^{\infty}(a x)^{\rho-1}(b y)^{\sigma-1} I_{p, q}^{m, n}\left[(a x)^{\lambda} \mid \begin{array}{l}
\left(\alpha_{1}, A_{1}, a_{1}\right), \ldots,\left(\alpha_{p}, A_{p}, a_{p}\right) \\
\left(\beta_{1}, B_{1}, b_{1}\right), \ldots,\left(\beta_{q}, B_{q}, b_{q}\right)
\end{array}\right] S_{n_{1}}^{m_{1}}\left[(a x)^{\delta_{1}}\right] \\
& I_{p^{\prime}, q^{\prime}}^{m^{\prime}, n^{\prime}}\left[(b y)^{\lambda^{\prime}} \mid \begin{array}{l}
\left(\alpha_{1}^{\prime}, A_{1}^{\prime}, a_{1}^{\prime}\right), \ldots,\left(\alpha_{p^{\prime}}^{\prime}, A_{p^{\prime}}^{\prime}, a_{p^{\prime}}^{\prime}\right) \\
\left(\beta_{1}^{\prime}, B_{1}^{\prime}, b_{1}^{\prime}\right), \ldots,\left(\beta_{q^{\prime}}^{\prime}, B_{q^{\prime}}, b_{q^{\prime}}\right)
\end{array}\right] S_{n_{2}}^{m_{2}}\left[(b y)^{\delta_{2}}\right] F(x, y) d x d y .
\end{aligned}
$$

Here, it is assumed that $h>0, g>0, \lambda>0, \lambda^{\prime}>0 ; \Delta(a, b)$ exists and belongs to $S_{2}$. Further assume

$$
\left|\arg a^{\lambda}\right|<\frac{\Omega \pi}{2}, \quad\left|\arg b^{\lambda^{\prime}}\right|<\frac{\Omega^{\prime} \pi}{2},
$$

where

$$
\begin{aligned}
\Omega & =\sum_{j=1}^{m}\left|b_{j} B_{j}\right|+\sum_{j=1}^{n}\left|a_{j} A_{j}\right|-\sum_{j=m+1}^{q}\left|b_{j} B_{j}\right|-\sum_{j=n+1}^{p}\left|a_{j} A_{j}\right|>0 \\
\Omega^{\prime} & =\sum_{j=1}^{m^{\prime}}\left|b_{j}^{\prime} B_{j}^{\prime}\right|+\sum_{j=1}^{n^{\prime}}\left|a_{j}^{\prime} A_{j}^{\prime}\right|-\sum_{j=m+1}^{q^{\prime}}\left|b_{j}^{\prime} B_{j}^{\prime}\right|-\sum_{j=n+1}^{p^{\prime}}\left|a_{j}^{\prime} A_{j}^{\prime}\right|>0 .
\end{aligned}
$$


4. Relationship between two - dimensional I - transform involving a general class of polynomials in terms of two -dimensional Saigo operator of Weyl type

For $\operatorname{Re}(u)>0, \operatorname{Re}(\eta)>0, g>0, \lambda>0$ and $\lambda^{\prime}>0$ then there holds the formula

$$
\begin{aligned}
& J_{x, \infty}^{u, v, w} J_{y, \infty}^{\eta, \beta, \alpha}\left[\Delta_{p, q, n_{1} ; p^{\prime}, q^{\prime} ; n_{2}}^{m, n, m_{1} ; m^{\prime}, m^{\prime}}\{F(x, y) ; \rho, \sigma ; a, b\}\right] \\
& =\int_{h}^{\infty} \int_{g}^{\infty}(a x)^{\rho-1}(b y)^{\sigma-1} I_{p+2, q+2}^{m+2, n}
\end{aligned}
$$

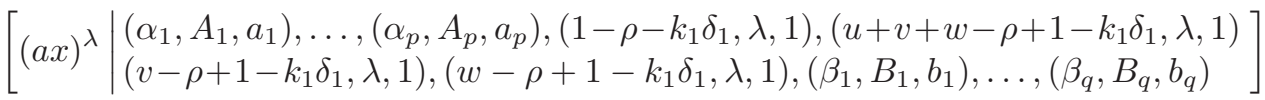

$$
\begin{aligned}
& S_{n_{1}}^{m_{1}}\left[(a x)^{\delta_{1}}\right] I_{p^{\prime}+2, q^{\prime}+2}^{m^{\prime}+2, n^{\prime}}
\end{aligned}
$$

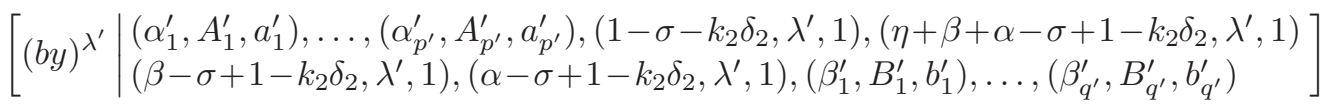

$$
\begin{aligned}
& S_{n_{2}}^{m_{2}}\left[(b y)^{\delta_{2}}\right] F(x, y) d x d y=\Delta_{1}(a, b) \text {, }
\end{aligned}
$$

where, it is supposed that $\Delta_{1}(a, b)$ exists and belongs to $S_{2}$ as well as $\lambda>0, \lambda^{\prime}>0$.

Proof. Let $\operatorname{Re}(u)>0, \operatorname{Re}(\eta)>0$ then in view of (16) and (21) we have

$J_{a, \infty}^{u, v, w} J_{b, \infty}^{\eta, \beta, \alpha}[\Delta(a, b)]$

$=\frac{a^{v} b^{\beta}}{\Gamma(u) \Gamma(\eta)} \int_{a}^{\infty} \int_{b}^{\infty}(t-a)^{u-1}(c-b)^{\eta-1} t^{-u-v} c^{-\eta-\beta}{ }_{2} F_{1}\left(u+v,-w ; u ; 1-\frac{a}{t}\right)_{2} F_{1}\left(\eta+\beta,-\alpha ; \eta ; 1-\frac{b}{c}\right)$

$\left\{\int_{h}^{\infty} \int_{g}^{\infty}(t x)^{\rho-1}(c y)^{\sigma-1} I_{p, q}^{m, n}\left[(t x)^{\lambda}\right] S_{n_{1}}^{m_{1}}\left[(t x)^{\delta_{1}}\right] I_{p^{\prime}, q^{\prime}}^{m^{\prime}, n^{\prime}}\left[(c y)^{\lambda^{\prime}}\right] S_{n_{2}}^{m_{2}}\left[(c y)^{\delta_{2}}\right] F(x, y) d x d y\right\} d t d c .(26)$

On interchanging the order of integration which is permissible due to absolute convergence of the integrals under consideration and on evaluating the $t$ - and $c$ - integrals through the integral formula Erdelyi [1]

$$
\int_{0}^{1} x^{\gamma-1}(1-x)_{2}^{\rho-1} F_{1}(\alpha, \beta ; \gamma ; x) d x=\frac{\Gamma(\gamma) \Gamma(\rho) \Gamma(\gamma+\rho-\alpha-\beta)}{\Gamma(\gamma+\rho-\alpha) \Gamma(\gamma+\rho-\beta)}
$$

for $\operatorname{Re}(\gamma)>0, \operatorname{Re}(\rho)>0, \operatorname{Re}(\gamma-\rho-\alpha-\beta)>0$.

For a little simplification L. H. S. of (26) become our required result (25).

\section{Special cases}

(i) By applying our results derived in (25) to the case of Hermite polynomials G. Szego

[4] and H. M. Srivastava [2] by setting

$$
S_{n}^{2}[x] \rightarrow x^{\frac{n}{2}} H_{n}\left(\frac{1}{2 \sqrt{x}}\right) \text { in which case } m=2, A_{n, k}=(-1)^{k}
$$


we have the following interesting consequence of the main result

$$
\begin{aligned}
& J_{a, \infty}^{u, v, w} J_{b, \infty}^{\eta, \beta, \alpha}\left[\Delta_{p, q, n_{1}, p^{\prime}, q^{\prime}, n_{2}}^{m, n, 2 ; m^{\prime}, n^{\prime} 2}\{F(x, y) ; \rho, \sigma ; a, b\}\right] \\
& =\int_{h}^{\infty} \int_{g}^{\infty}(a x)^{\rho+\frac{n_{1} \delta_{1}}{2}-1}(b y)^{\sigma+\frac{n_{2} \delta_{2}}{2}-1} I_{p+2, q+2}^{m+2, n} \\
& {\left[(a x)^{\lambda} \mid \begin{array}{l}
\left.\left(\alpha_{1}, A_{1}, a_{1}\right), \ldots,\left(\alpha_{p}, A_{p}, a_{p}\right),\left(1-\rho-k_{1} \delta_{1}, \lambda, 1\right),\left(u+v+w-\rho+1-k_{1} \delta_{1}, \lambda, 1\right)\right] \\
\left(v-\rho+1-k_{1} \delta_{1}, \lambda, 1\right),\left(w-\rho+1-k_{1} \delta_{1}, \lambda, 1\right),\left(\beta_{1}, B_{1}, b_{1}\right), \ldots,\left(\beta_{q}, B_{q}, b_{q}\right)
\end{array}\right]} \\
& H_{n_{1}}\left[\begin{array}{l}
\left.\frac{1}{2 \sqrt{(a x)^{\delta_{1}}}}\right] I_{p^{\prime}+2, q^{\prime}+2}^{m^{\prime}+2, n^{\prime}} \\
\left.(b y)^{\lambda^{\prime}} \mid \begin{array}{l}
\left(\alpha_{1}^{\prime}, A_{1}^{\prime}, a_{1}^{\prime}\right) \\
\left(\beta-\sigma+1-k_{2} \delta_{2}, \lambda^{\prime}, 1\right),\left(\alpha-\sigma \alpha_{p^{\prime}}^{\prime}, A_{p^{\prime}}^{\prime}, a_{p^{\prime}}^{\prime}\right),\left(1-\sigma-k_{2} \delta_{2}, \lambda^{\prime}, 1\right),\left(\eta+\beta+\alpha-\sigma+1-k_{2} \delta_{2}, \lambda^{\prime}, 1\right)
\end{array}\right] \\
H_{n_{2}}\left[\frac{1}{2 \sqrt{(b y)^{\delta_{2}}}}\right] F(x, y) d x d y,
\end{array}\right.
\end{aligned}
$$

(ii) For the Leaguerre polynomials (G. Szego [4] and H. M. Srivastava [2]) by setting $S_{n}^{1}[x] \rightarrow L_{n}^{\left(\alpha^{\prime}\right)}[x]$ in which case $m=1, A_{n, k}=\left(\begin{array}{c}n+\alpha^{\prime} \\ n\end{array}\right) \frac{1}{\left(\alpha^{\prime}+1\right)_{k}}$ and $(25)$ reduces to the following formulae

$$
\begin{aligned}
& J_{a, \infty}^{u, v, w} J_{b, \infty}^{\eta, \beta, \alpha}\left[\Delta_{p, q, n_{1}, p^{\prime}, q^{\prime}, n_{2}}^{m, n, 1 ; m^{\prime}, n^{\prime}}\{F(x, y) ; \rho, \sigma ; a, b\}\right] \\
& =\int_{h}^{\infty} \int_{g}^{\infty}(a x)^{\rho-1}(b y)^{\sigma-1} I_{p+2, q+2}^{m+2, n} \\
& {\left[(a x)^{\lambda} \mid \begin{array}{l}
\left(\alpha_{1}, A_{1}, a_{1}\right), \ldots,\left(\alpha_{p}, A_{p}, a_{p}\right),\left(1-\rho-k_{1} \delta_{1}, \lambda, 1\right),\left(u+v+w-\rho+1-k_{1} \delta_{1}, \lambda, 1\right) \\
\left(v-\rho+1-k_{1} \delta_{1}, \lambda, 1\right),\left(w-\rho+1-k_{1} \delta_{1}, \lambda, 1\right),\left(\beta_{1}, B_{1}, b_{1}\right), \ldots,\left(\beta_{q}, B_{q}, b_{q}\right)
\end{array}\right]} \\
& L_{n_{1}}^{\left(\alpha^{\prime}\right)}\left[(a x)^{\delta_{1}}\right] I_{p^{\prime}+2, q^{\prime}+2}^{m^{\prime}+2, n^{\prime}} \\
& {\left[(b y)^{\lambda^{\prime}} \mid \begin{array}{l}
\left(\alpha_{1}^{\prime}, A_{1}^{\prime}, a_{1}^{\prime}\right), \ldots,\left(\alpha_{p^{\prime}}^{\prime}, A_{p^{\prime}}^{\prime}, a_{p^{\prime}}^{\prime}\right),\left(1-\sigma-k_{2} \delta_{2}, \lambda^{\prime}, 1\right),\left(\eta+\beta+\alpha-\sigma+1-k_{2} \delta_{2}, \lambda^{\prime}, 1\right) \\
\left(\beta-\sigma+1-k_{2} \delta_{2}, \lambda^{\prime}, 1\right),\left(\alpha-\sigma+1-k_{2} \delta_{2}, \lambda^{\prime}, 1\right),\left(\beta_{1}^{\prime}, B_{1}^{\prime}, b_{1}^{\prime}\right), \ldots,\left(\beta_{q^{\prime}}^{\prime}, B_{q^{\prime}}^{\prime}, b_{q^{\prime}}^{\prime}\right)
\end{array}\right]} \\
& L_{n_{2}}^{\left(\alpha^{\prime}\right)}\left[(b y)^{\delta_{2}}\right] F(x, y) d x d y .
\end{aligned}
$$

(iii) For the Jacobi polynomials (G. Szego [4] and H. M. Srivastava [2]) by setting $S_{n}(x)=$ $P_{n}^{\left(\alpha^{\prime}, \beta^{\prime}\right)}(1-2 x)$ in which case $m=1$ and $A_{n, k}=\left(\begin{array}{c}n+\alpha^{\prime} \\ n\end{array}\right) \frac{\left(\alpha^{\prime}+\beta^{\prime}+n+1\right)_{k}}{\left(\alpha^{\prime}+1\right)_{k}}$ in $(25)$ reduces to following formulae

$$
\begin{aligned}
& J_{a, \infty}^{u, v, w} J_{b, \infty}^{\eta, \beta, \alpha}\left[\Delta_{p, q, n_{1}, p^{\prime}, q^{\prime}, n_{2}}^{m, n, 1 ; m^{\prime}, n^{\prime}}\{F(x, y) ; \rho, \sigma ; a, b\}\right] \\
& =\int_{h}^{\infty} \int_{g}^{\infty}(a x)^{\rho-1}(b y)^{\sigma-1} I_{p+2, q+2}^{m+2, n} \\
& {\left[\begin{array}{l}
\left.(a x)^{\lambda} \mid \begin{array}{l}
\left.\left(\alpha_{1}, A_{1}, a_{1}\right), \ldots,\left(\alpha_{p}, A_{p}, a_{p}\right),\left(1-\rho-k_{1} \delta_{1}, \lambda, 1\right),\left(u+v+w-\rho+1-k_{1} \delta_{1}, \lambda, 1\right)\right] \\
\left(v-\rho+1-k_{1} \delta_{1}, \lambda, 1\right),\left(w-\rho+1-k_{1} \delta_{1}, \lambda, 1\right),\left(\beta_{1}, B_{1}, b_{1}\right), \ldots,\left(\beta_{q}, B_{q}, b_{q}\right)
\end{array}\right] \\
P_{n_{1}}^{\left(\alpha^{\prime}, \beta^{\prime}\right)}\left[1-2(a x)^{\delta_{1}}\right] I_{p^{\prime}+2, q^{\prime}+2}^{m^{\prime}+2, n^{\prime}}
\end{array}\right.}
\end{aligned}
$$




$$
\begin{aligned}
& {\left[(b y)^{\lambda^{\prime}} \mid \begin{array}{l}
\left(\alpha_{1}^{\prime}, A_{1}^{\prime}, a_{1}^{\prime}\right), \ldots,\left(\alpha_{p^{\prime}}^{\prime}, A_{p^{\prime}}^{\prime}, a_{p^{\prime}}^{\prime}\right),\left(1-\sigma-k_{2} \delta_{2}, \lambda^{\prime}, 1\right),\left(\eta+\beta+\alpha-\sigma+1-k_{2} \delta_{2}, \lambda^{\prime}, 1\right) \\
\left(\beta-\sigma+1-k_{2} \delta_{2}, \lambda^{\prime}, 1\right),\left(\alpha-\sigma+1-k_{2} \delta_{2}, \lambda^{\prime}, 1\right),\left(\beta_{1}^{\prime}, B_{1}^{\prime}, b_{1}^{\prime}\right), \ldots,\left(\beta_{q^{\prime}}^{\prime}, B_{q^{\prime}}^{\prime}, b_{q^{\prime}}^{\prime}\right)
\end{array}\right]} \\
& \quad P_{n_{2}}^{\left(\alpha^{\prime}, \beta^{\prime}\right)}\left[1-2(b y)^{\delta_{2}}\right] F(x, y) d x d y,
\end{aligned}
$$

\section{Some known results}

(i) When the exponents $b_{j}(j=1, \ldots, m)$ and $a_{j}(j=n+1, \ldots, p)$ in (25) are unity, the I-function reduces to $\bar{H}$-function and we arrive at the result given by Chaurasia and Srivastava [12, pp.237-249].

(ii) On taking $a_{j}=b_{j}=1, n_{1}=n_{2}=0$ in (25), we arrive at the result obtained by Saigo, Saxena and Ram [10, pp.63-73].

(iii) For $a_{j}=b_{j}=1, n_{1}=n_{2}=0=w=\alpha$ in (25) we have a result, earlier proved by Saxena and Kiryakova [9, pp.133-140].

\section{Relationship between one-dimensional I-transform involving a general class} of polynomials in terms of one-dimensional Saigo operator of Weyl type

Definition. Let $\Delta(a)$ be the one-dimensional I-transform involving a general class of polynomials of $F(x)$ defined by

$$
\Delta(a)=\Delta_{p, q, n_{1}}^{m, n, m_{1}}[F(x) ; \rho, a]=\int_{h}^{\infty}(a x)^{\rho-1} I_{p, q}^{m, n}\left[(a x)^{\lambda}\right] S_{n_{1}}^{m_{1}}\left[(a x)^{\delta}\right] F(x) d x,
$$

provided that $\Delta(a)$ exists and belongs to $S_{1}$ where $\lambda>0,\left|\arg a^{\lambda}\right|<\frac{1}{2} \Omega \pi, F(x)=$ $F\left(d \sqrt{x^{2}-h^{2}}\right) H(x-h)$.

For $\operatorname{Re}(d)>0, h>0, \lambda>0$ and then the following formula

$$
\begin{aligned}
& \bar{J}_{a, \infty}^{u, v, w}\left[\Delta_{p, q, n_{1}}^{m, n, m_{1}}\{F(x) ; \rho, a\}\right]=\int_{h}^{\infty}(a x)^{\rho-1} I_{p+2, q+2}^{m+2, n} \\
& {\left[\begin{array}{l|l}
(a x)^{\lambda} & \begin{array}{l}
\left(\alpha_{1}, A_{1}, a_{1}\right), \ldots,\left(\alpha_{p}, A_{p}, a_{p}\right),(1-\rho-k \delta, \lambda, 1),(u+v+w+1-\rho-k \delta, \lambda, 1) \\
(v-\rho+1-k \delta, \lambda, 1),(w-\rho+1-k \delta, \lambda, 1),\left(\beta_{1}, B_{1}, b_{1}\right), \ldots,\left(\beta_{q}, B_{q}, b_{q}\right)
\end{array}
\end{array}\right]} \\
& S_{n_{1}}^{m_{1}}\left[(a x)^{\delta}\right] F(x) d x=\Delta_{1}(a),
\end{aligned}
$$

holds, provided that $\Delta_{1}(a)$ exists and belongs class $S_{1}$.

$$
\bar{J}_{a, \infty}^{u, v, w} f=\frac{a^{v}}{\Gamma(u)} \int_{0}^{\infty}(s-a)^{u-1} s^{-u-v}{ }_{2} F_{1}\left(u+v,-w ; u ; 1-\frac{a}{s}\right) f(s) d s .
$$




\section{Special cases}

(i) We take $v=0$ then $J_{a, \infty}^{u, 0, w} f=K_{a, \infty}^{u, w} f$ are the one-dimensional Erdelyi-Kober operator of fraction integration defined by (15) then the (31) reduces to the following formula

$$
\begin{aligned}
& K_{a, \infty}^{u, w}[\Delta(a)]=\int_{h}^{\infty}(a x)^{\rho-1} I_{p+1, q+1}^{m+1, n} \\
& \qquad \begin{array}{ll}
\left.(a x)^{\lambda} \mid \begin{array}{l}
\left(\alpha_{1}, A_{1}, a_{1}\right), \ldots,\left(\alpha_{p}, A_{p}, a_{p}\right),(u+v+w+1-\rho-k \delta, \lambda, 1) \\
(w-\rho+1-k \delta, \lambda, 1),\left(\beta_{1}, B_{1}, b_{1}\right), \ldots,\left(\beta_{q}, B_{q}, b_{q}\right)
\end{array}\right] \\
S_{n_{1}}^{m_{1}}\left[(a x)^{\delta}\right] F(x) d x,
\end{array}
\end{aligned}
$$

(ii) By applying our results derived in (31) to the case of Hermite polynomials G. Szego [4] and H. M. Srivastava [2] by setting $S_{n}^{2}[x] \rightarrow x^{(n / 2)} H_{n}\left(\frac{1}{2 \sqrt{x}}\right)$ in which case $m=2$, $A_{n, k}=(-1)^{k}$,

$$
\begin{aligned}
& \bar{J}_{a, \infty}^{u, v, w}\left[\Delta_{p, q, n_{1}}^{m, n, 2}\{F(x) ; \rho, a\}\right]=\int_{h}^{\infty}(a x)^{\rho+\frac{\delta_{n_{1}}}{2}-1} I_{p+2, q+2}^{m+2, n} \\
& \quad\left[\begin{array}{ll}
\left.(a x)^{\lambda} \mid \begin{array}{l}
\left(\alpha_{1}, A_{1}, a_{1}\right), \ldots,\left(\alpha_{p}, A_{p}, a_{p}\right),(1-\rho-k \delta, \lambda, 1),(u+v+w+1-\rho-k \delta, \lambda, 1) \\
(v-\rho+1-k \delta, \lambda, 1),(w-\rho+1-k \delta, \lambda, 1),\left(\beta_{1}, B_{1}, b_{1}\right), \ldots,\left(\beta_{q}, B_{q}, b_{q}\right)
\end{array}\right] \\
\quad H_{n_{1}}\left(\frac{1}{2 \sqrt{(a x)^{\delta}}}\right) F(x) d x,
\end{array}\right.
\end{aligned}
$$

(iii) For the Leaguerre polynomials (G. Szego [4] and H. M. Srivastava [2]) by setting $S_{n}^{1}[x] \rightarrow L_{n}^{\left(a^{\prime}\right)}[x]$ in which case $m=1, A_{n, k}=\left(\begin{array}{c}n+\alpha^{\prime} \\ n\end{array}\right) \frac{1}{\left(\alpha^{\prime}+1\right)_{k}}$ and (31) reduces to the following result

$$
\begin{aligned}
& \bar{J}_{a, \infty}^{u, v, w}\left[\Delta_{p, q, n_{1}}^{m, n, 1}\{F(x) ; \rho, a\}\right]=\int_{h}^{\infty}(a x)^{\rho-1} I_{p+2, q+2}^{m+2, n}
\end{aligned}
$$

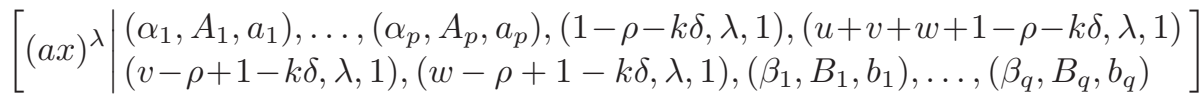

$$
\begin{aligned}
& L_{n_{1}}^{\left(\alpha^{\prime}\right)}\left[(a x)^{\delta}\right] F(x) d x .
\end{aligned}
$$

(iv) For the Jacobi polynomials (G. Szego [4] and H. M. Srivastava [2]) by setting $S_{n}(x)=P_{n}^{\left(\alpha^{\prime}, \beta^{\prime}\right)}(1-2 x)$ in which case $m=1$ and $A_{n, k}=\left(\begin{array}{c}n+\alpha^{\prime} \\ n\end{array}\right) \frac{\left(\alpha^{\prime}+\beta^{\prime}+n+1\right)_{k}}{\left(\alpha^{\prime}+1\right)_{k}}$ in (31) reduces to following formulae

$$
\begin{gathered}
\bar{J}_{a, \infty}^{u, v, w}\left[\Delta_{p, q, n_{1}}^{m, n, 1}\{F(x) ; \rho, a\}\right]=\int_{h}^{\infty}(a x)^{\rho-1} I_{p+2, q+2}^{m+2, n} \\
\qquad \begin{array}{ll}
\left.(a x)^{\lambda} \mid \begin{array}{l}
\left(\alpha_{1}, A_{1}, a_{1}\right), \ldots,\left(\alpha_{p}, A_{p}, a_{p}\right),(1-\rho-k \delta, \lambda, 1),(u+v+w+1-\rho-k \delta, \lambda, 1) \\
(v-\rho+1-k \delta, \lambda, 1),(w-\rho+1-k \delta, \lambda, 1),\left(\beta_{1}, B_{1}, b_{1}\right), \ldots,\left(\beta_{q}, B_{q}, b_{q}\right)
\end{array}\right] \\
P_{\left.n_{1}, \alpha^{\prime}, \beta^{\prime}\right)}\left[1-2(a x)^{\delta}\right] F(x) d x .
\end{array}
\end{gathered}
$$




\section{Some Interesting Known Results}

(i) If we take $b_{j}(j=1, \ldots, m)=1$ and $a_{j}(j=n+1, \ldots, p)=1$, (31) reduces to the result obtained by Chaurasia and Srivastava [12, pp.237-249].

(ii) Taking $a_{j}=b_{j}=1, n_{1}=n_{2}=0$ in (31) we arrive at the result obtained by Saigo, Saxena and Ram [10, pp.63-73].

(iii) On taking $a_{j}=b_{j}=1, n_{1}=n_{2}=0$ in (33), we get the result earlier proved by Saigo, Saxena and Ram [10, pp.63-73].

\section{Acknowledgement}

The authers are grateful to Professor H. M. Srivastava (University of Victoria, Canada) for his kind help and suggestions in the preparation of this paper.

\section{References}

[1] A. Erdelyi, W. Magnus, F. Oberhettinger and F. G. Tricomi, Table of Integral Transforms, Vol. 2, McGraw-Hill, New York-Toronto-London, 1954.

[2] H. M. Srivastava, A Contour Integral Involving Fox's H-function, Indian, J. Math. 14 (1972)

[3] K. S. Miller, The Weyl Fractional Calculus, Fractional Calculus and Its Applications, Lecturer Notes in Math., Vol. 457, Springer, Berlin-Heidelberg-New York, 1975, 80-89.

[4] G. Szego, Orthogonal Polynomials, Amer. Math. Soc. Colloq. Publ. 23 Fourth Edition, Amer. Math. Soc. Providence, Rhode Island, 1975.

[5] M. Saigo, A Remark on integral operators involving the Gauss's hypergeometric functions, Math. Rep. College General Ed. Kyushu Uni., 11(1978), 135-143

[6] M. Saigo, Certain boundary value problem for the Euler-Darbooux equation, Math. Japon, 24(1979), 377-385.

[7] H. M. Srivastava and N. P. Singh, The integration of certain products of the multivariable $H$-function with a general class of polynomials rend, Circ. Math. Palermo (2) 32(1983), $157-187$.

[8] R. K. Saxena and J. Ram, On the two-dimensional Whittaker transform, SERDICA Bulg. Math. Publ., 16(1990), 27-30.

[9] R. K. Saxena and V. S. Kiryakova, On the two-dimensional H-transforms in terms of Erdelyi-Kober operators, Math. Balkanica, 6(1992), 133-140.

[10] M. Saigo, R. K. Saxena and J. Ram, On the two- dimensional Weyl fractional calculus, Associated with Two dimensional H-transforms, Journal of Fractional Calculus (ISSN 09185402) 8(1995), 63-73, Descartes Press.

[11] A. K. Rathie, A new generalization of generalized hypergeometric functions, Le Mathematiche, 52(1997), 297-310.

[12] V.B.L. Churasia and A. Srivastava, Two -dimensional generalized Weyl fractional calculus pertaining to two-dimensional - transforms, Tamkang Journal of Mathematics, Taiwan $37(2000), 237-249$. 
Department of Mathematics, University of Rajasthan, Jaipur-302055, India.

E-mail: agnivasu@yahoo.co.in 